STRUCTURAL BIOLOGY COMMUNICATIONS

ISSN 2053-230X

\section{Crystal structure of yeast monothiol glutaredoxin Grx6 in complex with a glutathione-coordinated [2Fe-2S] cluster}

\author{
Mohnad Abdalla, Ya-Nan Dai, ${ }^{*}$ Chang-Biao Chi, Wang Cheng, Dong-Dong Cao, \\ Kang Zhou, Wafa Ali, Yuxing Chen and Cong-Zhao Zhou*
}

Hefei National Laboratory for Physical Sciences at the Microscale and School of Life Sciences, University of Science and Technology of China, Hefei, Anhui 230027, People's Republic of China. *Correspondence e-mail:

daiyanan@mail.ustc.edu.cn, zcz@ustc.edu.cn

Edited by M. L. Pusey, University of Alabama, USA

Keywords: glutaredoxin; iron-sulfur cluster; Saccharomyces cerevisiae; crystal structure; oxidoreductase.

PDB reference: Grx6, complex with a glutathione-coordinated [2Fe-2S] cluster, $5 \mathrm{j} 3 \mathrm{r}$

Supporting information: this article has supporting information at journals.iucr.org/f

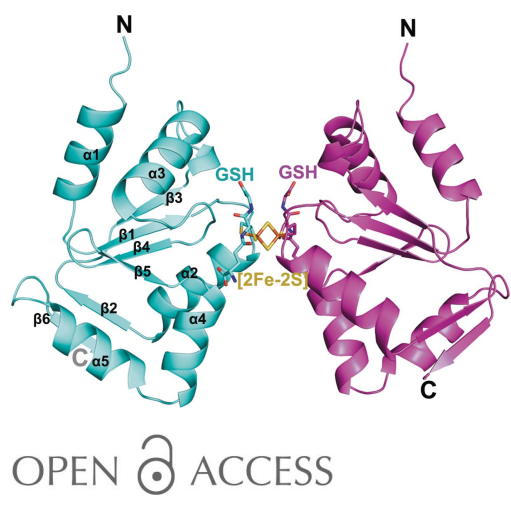

Glutaredoxins (Grxs) constitute a superfamily of proteins that perform diverse biological functions. The Saccharomyces cerevisiae glutaredoxin Grx6 not only serves as a glutathione (GSH)-dependent oxidoreductase and as a GSH transferase, but also as an essential [2Fe-2S]-binding protein. Here, the dimeric structure of the C-terminal domain of Grx6 (holo Grx6C), bridged by one [2Fe-2S] cluster coordinated by the active-site Cys136 and two external GSH molecules, is reported. Structural comparison combined with multiple-sequence alignment demonstrated that holo Grx6C is similar to the [2Fe-2S] clusterincorporated dithiol Grxs, which share a highly conserved [2Fe-2S] clusterbinding pattern and dimeric conformation that is distinct from the previously identified $[2 \mathrm{Fe}-2 \mathrm{~S}]$ cluster-ligated monothiol Grxs.

\section{Introduction}

Glutaredoxins (Grxs) are thioredoxin-fold oxidoreductases that utilize glutathione (GSH) as the reductant to maintain redox homeostasis in all kingdoms of life (Li, 2014). According to their active-site sequences, Grxs were initially classified as classic dithiol Grxs with a $\mathrm{C} X X \mathrm{C}$ motif and monothiol Grxs containing a $\mathrm{C} X X \mathrm{~S}$ motif. A subsequent thorough comparative genomic analysis identified six families of Grxs, among which classes I and II are the most widespread (Rouhier et al., 2010). Class I proteins contain both dithiol (CP/G/S/FYC, 2-C-Grxs) and monothiol (CP/SYS, 1-C-Grxs) Grxs. Class II proteins are 1-C-Grxs that harbour a strict CGFS motif. Generally, 2-C-Grxs contribute greatly to reducing proteinGSH mixed disulfides and intermolecular or intramolecular disulfides, whereas most 1-C-Grxs show decreased activities in catalyzing thiol-disulfide exchange reactions but adopt more specialized functions in cells (Manta et al., 2013). For instance, class II 1-C-Grxs can coordinate [2Fe-2S] iron-sulfur clusters (ISCs), evolutionarily ancient prosthetic groups that are necessary to maintain essential life processes (Zhang, 2015). Interestingly, several 2-C-Grxs such as human Grx2 (CSYC motif) and poplar GrxC1 (CGYC motif) have also been found to bind ISC (Comini et al., 2013).

The yeast Saccharomyces cerevisiae encodes eight Grxs (Grx1-Grx8) with diverse subcellular localizations and functions. In detail, Grx1 and Grx2 are cytosolic 2-C-Grxs harbouring a CPYC motif that are responsible for resistance to various hydroperoxides (Collinson et al., 2002). Grx3, Grx4 and Grx5 are all 1-C-Grxs containing a CGFS motif for ISC 
coordination. Grx3 and Grx4 take part in regulation of iron uptake in the nucleus by interacting with the transcriptional activator Aft1, whereas Grx5 is required for ISC biogenesis in mitochondria (Tamayo et al., 2016). Grx6 and Grx7 belong to the class I 1-C-Grxs with a CSYS and a CPYS motif, respectively; they are integral components of ER/Golgi membranes and are associated with the early secretory pathway (Lee $e t a l$., 2007). Grx8 is a cytosolic 2-C-Grx with a CPDC motif and has a much lower GSH-dependent oxidoreductase activity (Tang et al., 2014).

We have been working on yeast Grxs to reveal their structural and biochemical characteristics ( $\mathrm{Li}, 2014$; Luo et al., 2010; Tang et al., 2014). To date, we have solved the crystal structures of yeast Grx1 (PDB entries 3c1r and 3c1s; Yu et al., 2008), Grx2 (PDB entries 3ctf and 3ctg; Li et al., 2010), Grx5 (PDB entry 3gx8; Y. Wang, Y.-X. He, J. Yu, Y. Xiong, Y. Chen

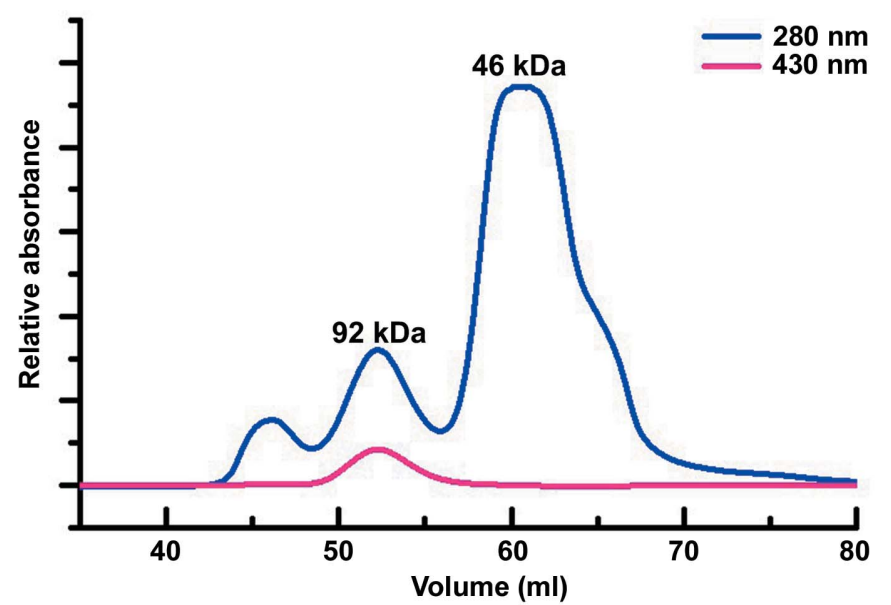

(a)

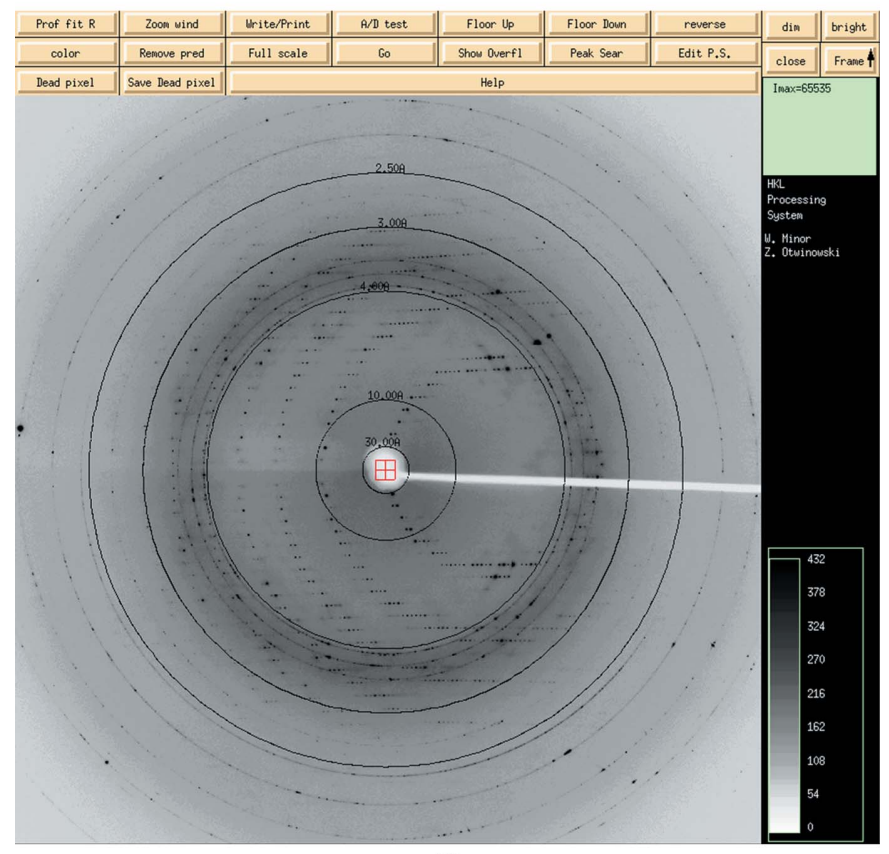

(b)

Figure 1

(a) Gel-filtration chromatography of full-length Grx6 and $(b)$ a representative diffraction image for data collection from full-length Grx6.
\& C.-Z. Zhou, unpublished work), the C-terminal domain of Grx6 (PDB entry 314n; Luo et al., 2010) and Grx8 (PDB entry 2m80; Tang et al., 2014). In contrast to the other Grxs, Grx6 consists of three segments: a putative signal peptide (Met1-Ile36), an N-terminal domain (Lys37-Thr110) and a C-terminal Grx domain (Lys111-Asn231, designated Grx6C). It is worth mentioning that Grx6, which is more similar in activity to 2-C-Grxs than to other 1-C-Grxs, has a lower glutathione disulfide reductase activity but a higher glutathione transferase activity compared with yeast Grx1 (Luo et $a l ., 2010)$. It has been suggested that Grx6 could modulate the glutathionylation state of target proteins at the ER/Golgi lumen and consequently regulate the glutathione redox balance (Puigpinós et al., 2015). Moreover, Grx6 is capable of bridging ISCs through its $\mathrm{C}$-terminal domain, which possesses a unique motif of a two-strand antiparallel $\beta$-sheet beyond the typical Grx fold. Although the in vivo function of Grx6 binding to ISC remains to be elucidated, it is strongly indicated that Grx6 could serve as a trifunctional Grx in the cell, defining it as the head of a novel Grx subfamily (Luo et al., 2010).

However, the structural features of the coordination of the ISC in Grx6 are still unknown. Here, we report the dimeric crystal structure of a $[2 \mathrm{Fe}-2 \mathrm{~S}]$ cluster-bound form of Grx6C (designated holo Grx6C) at $2.46 \AA$ resolution. Holo Grx6C comprises a subunit-bridging $[2 \mathrm{Fe}-2 \mathrm{~S}]$ cluster that is ligated by the catalytic Cys136 residues of each subunit and two external GSH molecules. It adopts a highly similar ISC-binding pattern and dimeric conformation to the holo structure of human Grx2 (PDB entry 2ht9; Johansson et al., 2007), but differs from the monothiol human Grx5 (CGFS motif; PDB entry 2wul; Johansson et al., 2011), which displays a more extended conformation. Structure-based multiple-sequence alignment implied that holo Grx6C is more similar to ISC-linked 2-CGrxs compared with class II 1-C-Grxs. In summary, yeast Grx6 is probably not only an oxidoreductase, but is also an ISC storage or delivery protein in the cell.

\section{Materials and methods}

\subsection{Cloning, expression and purification of Grx6}

The coding region of Grx6/YDL010W without the signal peptide (residues Lys37-Asn231, designated Grx6) was PCRamplified from $S$. cerevisiae S288c genomic DNA and cloned into the pET-22b(+) vector (Novagen) with a C-terminal $\mathrm{His}_{6}$ tag. The recombinant plasmid was transformed into Escherichia coli strain BL21 (DE3) (Novagen) cultured in LB culture medium $(10 \mathrm{~g} \mathrm{NaCl}, 10 \mathrm{~g}$ Bacto tryptone and $5 \mathrm{~g}$ yeast extract per litre) containing $50 \mathrm{mg} \mathrm{ml}^{-1}$ ampicillin at $37^{\circ} \mathrm{C}$. The expression of recombinant protein was induced using $0.02 \mathrm{mM}$ IPTG at $16^{\circ} \mathrm{C}$ for $20 \mathrm{~h}$ after the cells had grown to an $\mathrm{OD}_{600 \mathrm{~nm}}$ of 0.8 . Cells were collected by centrifugation and resuspended in buffer $(20 \mathrm{~m} M \mathrm{NaCl}, 50 \mathrm{~m} M$ Tris- $\mathrm{HCl} \mathrm{pH} 8.0)$. After $20 \mathrm{~min}$ of sonication followed by centrifugation at $12000 \mathrm{~g}$ for $25 \mathrm{~min}$, the supernatant was pooled and loaded onto an NiNTA column (GE Healthcare). The target protein was eluted 
with $250 \mathrm{~m} M$ imidazole and loaded onto a HiLoad 16/60 Superdex 75 column pre-equilibrated with buffer $(20 \mathrm{mM}$ $\mathrm{NaCl}, 50 \mathrm{~m} M$ Tris-HCl pH 8.0, $2 \mathrm{~m} M$ EDTA, $10 \mathrm{~m} M$ GSH). Similar to a previous report (Luo et al., 2010), gel-filtration chromatography of Grx6 showed separated peaks representing distinctive oligomeric states (Fig. 1a). The protein from the peak with a strong absorbance at $430 \mathrm{~nm}$ owing to the presence of the ISC was pooled and concentrated to $10 \mathrm{mg} \mathrm{ml}^{-1}$ for crystallization screening.

\subsection{Crystallization, data collection and processing}

Grx6 crystals were grown at $14^{\circ} \mathrm{C}$ by the sitting-drop vapour-diffusion method using $1.0 \mu \mathrm{l}$ protein sample, prepared as described above, and an equal volume of reservoir solution (0.1 $M$ HEPES sodium $\mathrm{pH}$ 7.5, $0.8 M$ potassium sodium tartrate tetrahydrate). After about $12 \mathrm{~h}$, diamond-shaped crystals appeared. A single crystal was transferred to cryoprotectant (reservoir solution supplemented with 20\% glycerol) and flash-cooled with liquid nitrogen. X-ray diffraction data were collected at $100 \mathrm{~K}$ in a liquid-nitrogen stream on beamline $17 \mathrm{U}$ at the Shanghai Synchrotron Radiation Facility using an ADSC Q315r CCD detector (Area Detector Systems Corporation). The data sets were processed and scaled using $H K L-2000$ (Otwinowski \& Minor, 1997).

\subsection{Structure determination and refinement}

The structure of holo Grx6C was determined by molecular replacement with MOLREP in CCP4 (Winn et al., 2011) using the structure of yeast GSH-Grx6C (PDB entry 314n; Luo et al., 2010) as a search model. Refinement was performed with REFMAC5 (Murshudov et al., 2011) and the model was rebuilt interactively using Coot (Emsley et al., 2010). Water molecules and ligands were then placed into the electron-density map. The structure was finally refined to $2.46 \AA$ resolution with an $R$ factor of $19.6 \%$ and an $R_{\text {free }}$ of $23.0 \%$. An overall assessment of model quality was performed using MolProbity (Chen et al., 2010) and PROCHECK (Laskowski et al., 1993). Datacollection and refinement statistics are listed in Table 1. All structural figures were prepared with PyMOL (Schrödinger).

\section{Results and discussion}

\subsection{Overall structure of holo Grx6C}

Gel-filtration chromatography showed that full-length Grx6 eluted as three peaks (Fig. 1a). The first peak corresponds to aggregated protein, and the other two correspond to apparent molecular masses of $46 \mathrm{kDa}$ (dimer) and $92 \mathrm{kDa}$ (tetramer). Protein from the peak with an absorbance at $430 \mathrm{~nm}$, owing to the presence of the ISC, was used for crystallization. We obtained diamond-shaped crystals and they diffracted well. A representative diffraction image from the data collection is shown in Fig. 1(b). However, after structure solution only Grx6C could be built into the final model. An electrophoretic assay of the crystals confirmed that the protein had not been degraded during crystallization, indicating high flexibility of the N-terminal domain. Each asymmetric unit of the crystal
Table 1

Crystal parameters, data collection and structure refinement of holo Grx6C.

Values in parentheses are for the highest resolution bin.

\begin{tabular}{|c|c|}
\hline \multicolumn{2}{|l|}{ Data collection } \\
\hline Space group & $P 4_{3} 2_{1} 2$ \\
\hline Unit-cell parameters $\left(\AA{ }^{\circ},{ }^{\circ}\right)$ & $\begin{array}{c}a=b=58.657, c=161.763 \\
\alpha=\beta=\gamma=90.00\end{array}$ \\
\hline Resolution range $(\AA)$ & $50.00-2.45(2.54-2.45)$ \\
\hline Wilson $B$ factor $\left(\AA^{2}\right)$ & 45.0 \\
\hline Unique reflections & 10929 (1055) \\
\hline Completeness (\%) & $99.4(99.5)$ \\
\hline$\langle I / \sigma(I)\rangle$ & $23.503(8.455)$ \\
\hline$R_{\text {merge }} \dagger(\%)$ & $8.1(37.9)$ \\
\hline Average multiplicity & $12.6(13.2)$ \\
\hline \multicolumn{2}{|l|}{ Structure refinement } \\
\hline Resolution range $(\AA)$ & $28.96-2.46$ \\
\hline$R$ factor $\$ / R_{\text {free }} \S(\%)$ & 19.6/23.0 \\
\hline No. of protein atoms & 918 \\
\hline No. of ligands & 2 \\
\hline No. of water molecules & 53 \\
\hline R.m.s.d. $\mid$, bond lengths $(\AA)$ & 0.009 \\
\hline R.m.s.d., bond angles $\left({ }^{\circ}\right)$ & 1.368 \\
\hline \multicolumn{2}{|l|}{ Mean $B$ factors $\left(\AA^{2}\right)$} \\
\hline Protein & 45.1 \\
\hline Ligand & 42.6 \\
\hline Water & 50.5 \\
\hline \multicolumn{2}{|l|}{ Ramachandran plot } \\
\hline Poor rotamers $(\%)$ & 0 \\
\hline Most favoured (\%) & 99.11 \\
\hline Additionally allowed (\%) & 0.89 \\
\hline Outliers (\%) & 0 \\
\hline PDB entry & $5 \mathrm{j} 3 \mathrm{r}$ \\
\hline
\end{tabular}

$\dagger R_{\text {merge }}=\sum_{h k l} \sum_{i}\left|I_{i}(h k l)-\langle I(h k l)\rangle\right| / \sum_{h k l} \sum_{i} I_{i}(h k l)$, where $I_{i}(h k l)$ is the intensity of an observation and $\langle I(h k l)\rangle$ is the mean value for the unique reflection; summations are over all reflections. $\neq R$ factor $=\sum_{h k l}|| F_{\text {obs }}|-| F_{\text {calc }}|| / \sum_{h k l}\left|F_{\text {obs }}\right|$, where $F_{\text {obs }}$ and $F_{\text {calc }}$ are the observed and calculated structure-factor amplitudes, respectively. $\& R_{\text {free }}$ was calculated with $5 \%$ of the data, which were excluded from the refinement. I Rootmean-square deviation from ideal values.

contained one molecule of Grx6C. Analysis of the crystal packing suggests one [2Fe-2S] cluster-bridged homodimer with a buried surface area of $196 \AA^{2}$ (Fig. 2a). At a closer viewpoint on the dimeric interface, the $[2 \mathrm{Fe}-2 \mathrm{~S}]$ cluster is coordinated by the active-site Cys 136 from each monomer in addition to two GSH molecules. Compared with the typical Grx fold, Grx6C comprises a two-stranded antiparallel $\beta$-sheet ( $\beta 2$ and $\beta 6$ ) in addition to a central four-stranded antiparallel $\beta$-sheet ( $\beta 1$ and $\beta 3-\beta 5$ ) surrounded by five $\alpha$-helices $(\alpha 1-\alpha 5)$. The overall structures of holo Grx6C and GSH-Grx6C are quite similar, with a root-mean-square deviation of $0.28 \AA$ over $100 \mathrm{C}^{\alpha}$ atoms (Fig. $2 b$ ).

\section{2. $\mathrm{GSH} /[2 \mathrm{Fe}-2 \mathrm{~S}]-$ binding site}

The $[2 \mathrm{Fe}-2 \mathrm{~S}]$ cluster and two GSH models fit well in the electron-density map (Fig. 2c). The pair of Fe atoms in the [2Fe-2S] cluster are coordinated by the $\mathrm{S}$ atoms of Cys136 from both subunits and two GSH molecules (Fig. 2c). It is noted that the hydrophobic side chains of Tyr138 from both subunits contribute to the formation of the dimeric interface and help stabilize the cluster by reducing the solvent accessibility (Fig. 2d).

GSH molecules are mainly fixed by a network of hydrogen bonds. Taking subunit $A$ for instance, the GSH-binding groove mainly consists of the following parts: residues Asn196 and 
Glu197 from helix $\alpha 4$, Thr182 and Vla183 from the loop between $\alpha 3$ and $\beta 4$, Thr135 from the loop connecting $\beta 1$ and $\alpha 2$, and a patch composed of Lys133 and Gln171 (Fig. 2d). In detail, Asn196 and Glu197 assist in the formation and orientation of helix $\alpha 4$, which is crucial for the proper positioning of GSH. The $\mathrm{N}^{\alpha}$ atoms of both Asn196 and Glu197 form hydrogen bonds to the carboxyl group of the glutamyl moiety, which is further stabilized by the side chain of Thr182 via water-mediated (Wat1) hydrogen bonds. The cysteinyl carboxyl $\mathrm{O}$ atom is fixed by a hydrogen bond to the $\mathrm{N}^{\alpha}$ atom of Val183. The $\mathrm{N}^{\alpha}$ atom of the glycinyl moiety is stabilized by the main-chain $\mathrm{O}$ atom of Thr135 from the neighbouring subunit, whereas the carboxyl group of the glycinyl moiety is fixed by polar interactions with side chains of Lys133 and Gln171.

\subsection{Comparison of Grx6 with ISC-ligated Grxs}

To date, five structures of ISC-ligated Grxs have been deposited in the PDB, including three class I 2-C-Grxs and two class II 1-C-Grxs. The 2-C-Grxs are human Grx2, poplar GrxC1 (PDB entry 2e7p; Rouhier et al., 2007) and A. thaliana GrxC5 (PDB entry 3rhc; Couturier et al., 2011), whereas the 1-C-Grxs include human Grx5 and E. coli Grx4 (PDB entry 2 wci; Iwema et al., 2009). Structural comparison demonstrated

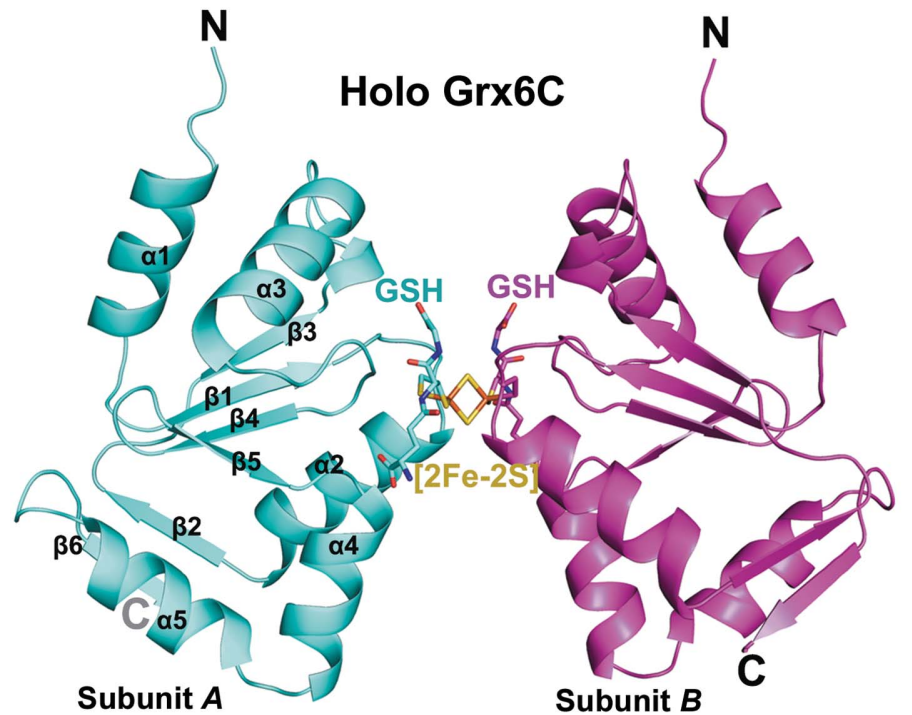

(a)

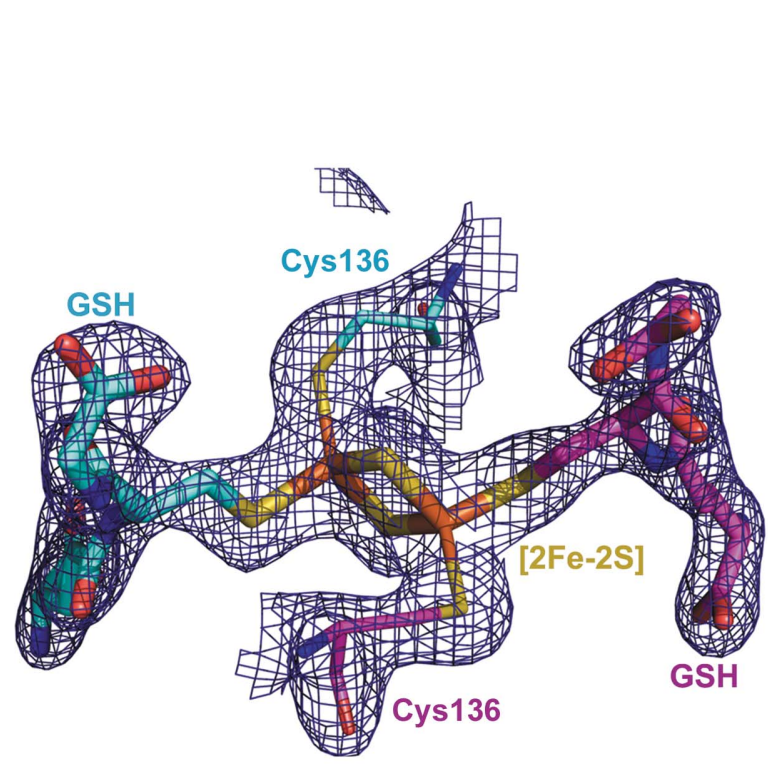

(c)

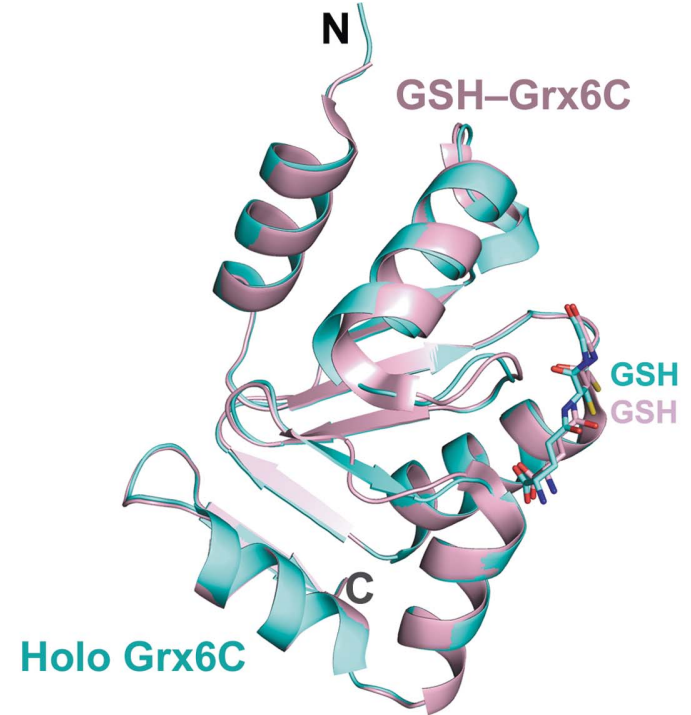

(b)
Figure 2

Crystal structure of yeast Grx6C bound to GSH and a [2Fe-2S] cluster. (a) The dimeric structure. Subunits $A$ and $B$ are coloured cyan and magenta, respectively. Secondary structures are labelled in subunit $A$. Two GSH molecules and the bridged [2Fe-2S] cluster are shown as sticks. $\mathrm{S}$ atoms are coloured yellow and Fe atoms brown. (b) Comparison of the overall structure between subunit $A$ (cyan) of holo Grx6C and GSH-Grx6C (light magenta). (c) $\sigma_{\mathrm{A}}$-weighted $2 F_{\mathrm{o}}-F_{\mathrm{c}}$ density map of Cys136 and the GSH-linked [2Fe-2S]. (d) Stereo representation of the [2Fe-2S] cluster coordination and GSH-Grx6C interactions. Two GSH molecules and the bridged [2Fe-2S] cluster are shown as bold sticks. Residues involved in GSH interaction are shown as sticks. Polar interactions are indicated by dashed lines. 


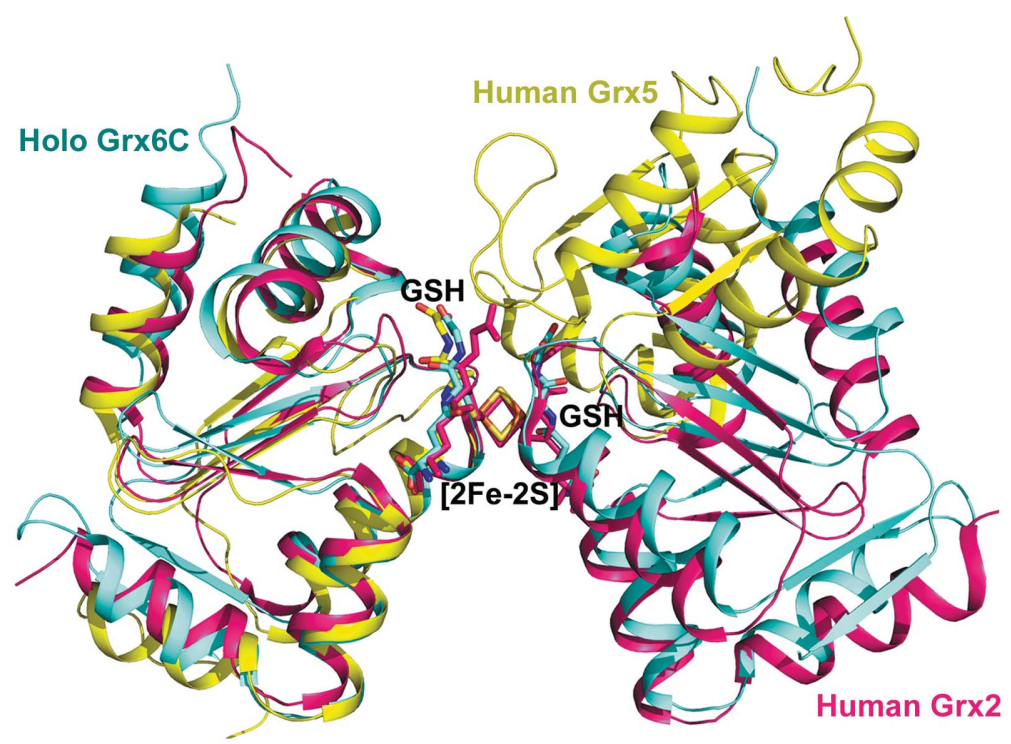

(a)
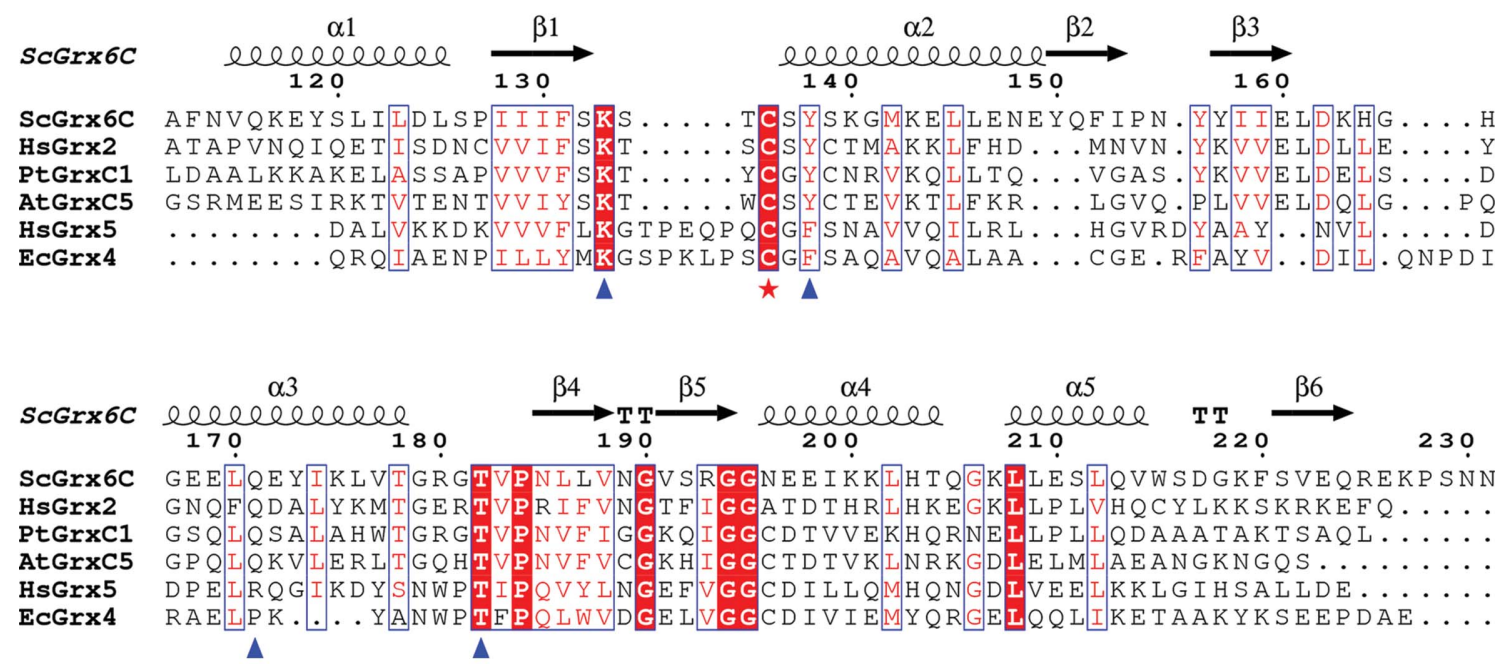

(b)

Figure 3

Comparison of structures of ISC-ligated Grxs. (a) Superposition of holo Grx6C (cyan) with human Grx2 (pink) and human Grx5 (yellow). (b) Multiple sequence alignment of solved structures of ISC-ligated Grxs. The active-site Cys136 and residues involved in GSH and [2Fe-2S] stabilization are indicated with a red star and blue triangles, respectively. The multiple sequence alignment was performed using ESPript (http://espript.ibcp.fr/ESPript/ cgi-bin/ESPript.cgi). The sequences are Saccharomyces cerevisiae Grx6C (ScGrx6C), Homo sapiens Grx2 $($ HsGrx2), Populus tremula $\times$ Populus tremuloides GrxC1 (PtGrxC1), Arabidopsis thaliana GrxC5 (AtGrxC5), Homo sapiens Grx5 (HsGrx5) and Escherichia coli Grx4 (EcGrx4).

that holo Grx6C displays a dimeric conformation that is highly similar to that of human Grx2 but different from human Grx5 (Fig. 3a), in which the conformation is more extended with more extensive intersubunit interactions. In addition, among the residues whose side chains stabilize GSH and the [2Fe-2S] cluster in holo Grx6C, Lys133 and Thr182 are highly conserved, whereas Tyr138 and Asn171 are more conserved in ISC-bound 2-C-Grxs than in 1-C-Grxs (Fig. 3b). Taken together, Grx6 is more similar to ISC-linked 2-C-Grxs in both primary sequence and three-dimensional structure, compared with 1-C-Grxs harbouring the CGFS motif. The ISC has been suggested to function as a redox sensor for the activation of Grxs (Lillig et al., 2005). For instance, human Grx2 becomes active upon loss of the [2Fe-2S] cluster in vitro by GSSG or in vivo by a decrease in the GSH:GSSG ratio (Lillig et al., 2005).
Therefore, Grx6 could also serve as an oxidoreductase regulated by the redox sensing of the incorporated ISC, in addition to the possibility of it being an ISC storage/delivery protein. Furthermore, the presence of a unique $\mathrm{N}$-terminal domain indicates increased complexity of the cellular roles of Grx6, which calls for further functional explorations in the future. Nevertheless, the structural insights from holo Grx6C shed light on the diverse functions of yeast Grx6 to some degree.

\section{Acknowledgements}

We thank the beamline staff of the Shanghai Synchrotron Radiation Facility (SSRF) for technical help during X-ray data collection. This work was supported by Project 2012CB911002 from the Ministry of Science and Technology of China and the 
CAS-TWAS President's PhD Fellowship to MA and WA. All authors declare no conflicts of interest.

\section{References}

Chen, V. B., Arendall, W. B., Headd, J. J., Keedy, D. A., Immormino, R. M., Kapral, G. J., Murray, L. W., Richardson, J. S. \& Richardson, D. C. (2010). Acta Cryst. D66, 12-21.

Collinson, E. J., Wheeler, G. L., Garrido, E. O., Avery, A. M., Avery, S. V. \& Grant, C. M. (2002). J. Biol. Chem. 277, 16712-16717.

Comini, M. A., Krauth-Siegel, R. L. \& Bellanda, M. (2013). Antioxid. Redox Signal. 19, 708-722.

Couturier, J., Stroher, E., Albetel, A. N., Roret, T., Muthuramalingam, M., Tarrago, L., Seidel, T., Tsan, P., Jacquot, J.-P., Johnson, M. K., Dietz, K. J., Didierjean, C. \& Rouhier, N. (2011). J. Biol. Chem. 286, 27515-27527.

Emsley, P., Lohkamp, B., Scott, W. G. \& Cowtan, K. (2010). Acta Cryst. D66, 486-501.

Iwema, T., Picciocchi, A., Traore, D. A. K., Ferrer, J.-L., Chauvat, F. \& Jacquamet, L. (2009). Biochemistry, 48, 6041-6043.

Johansson, C., Kavanagh, K. L., Gileadi, O. \& Oppermann, U. (2007). J. Biol. Chem. 282, 3077-3082.

Johansson, C., Roos, A. K., Montano, S. J., Sengupta, R., Filippakopoulos, P., Guo, K., von Delft, F., Holmgren, A., Oppermann, U. \& Kavanagh, K. L. (2011). Biochem. J. 433, 303-311.

Laskowski, R. A., MacArthur, M. W., Moss, D. S. \& Thornton, J. M. (1993). J. Appl. Cryst. 26, 283-291.

Lee, J.-H., Kim, K., Park, E.-H., Ahn, K. \& Lim, C.-J. (2007). Mol. Cells, 24, 316-322.

Li, S. (2014). Plants, 3, 559-582.
Li, W.-F., Yu, J., Ma, X.-X., Teng, Y.-B., Luo, M., Tang, Y.-J. \& Zhou, C.-Z. (2010). Biochim. Biophys. Acta, 1804, 1542-1547.

Lillig, C. H., Berndt, C., Vergnolle, O., Lönn, M. E., Hudemann, C., Bill, E. \& Holmgren, A. (2005). Proc. Natl Acad. Sci. USA, 102, 8168-8173.

Luo, M., Jiang, Y.-L., Ma, X.-X., Tang, Y.-J., He, Y.-X., Yu, J., Zhang, R.-G., Chen, Y. \& Zhou, C.-Z. (2010). J. Mol. Biol. 398, 614622.

Manta, B., Pavan, C., Sturlese, M., Medeiros, A., Crispo, M., Berndt, C., Krauth-Siegel, R. L., Bellanda, M. \& Comini, M. A. (2013). Antioxid. Redox Signal. 19, 665-682.

Murshudov, G. N., Skubák, P., Lebedev, A. A., Pannu, N. S., Steiner, R. A., Nicholls, R. A., Winn, M. D., Long, F. \& Vagin, A. A. (2011). Acta Cryst. D67, 355-367.

Otwinowski, Z. \& Minor, W. (1997). Methods Enzymol. 276, 307-326.

Puigpinós, J., Casas, C. \& Herrero, E. (2015). Mol. Biol. Cell, 26, 104-116.

Rouhier, N., Couturier, J., Johnson, M. K. \& Jacquot, J.-P. (2010). Trends Biochem. Sci. 35, 43-52.

Rouhier, N., Unno, H., Bandyopadhyay, S., Masip, L., Kim, S.-K., Hirasawa, M., Gualberto, J. M., Lattard, V., Kusunoki, M., Knaff, D. B., Georgiou, G., Hase, T., Johnson, M. K. \& Jacquot, J.-P. (2007). Proc. Natl Acad. Sci. USA, 104, 7379-7384.

Tamayo, E., Benabdellah, K. \& Ferrol, N. (2016). PLoS One, 11, e0149606.

Tang, Y., Zhang, J., Yu, J., Xu, L., Wu, J., Zhou, C.-Z. \& Shi, Y. (2014). Biochemistry, 53, 2185-2196.

Winn, M. D. et al. (2011). Acta Cryst. D67, 235-242.

Yu, J., Zhang, N.-N., Yin, P.-D., Cui, P.-X. \& Zhou, C.-Z. (2008). Proteins, 72, 1077-1083

Zhang, C. (2015). Genome Integr. 6, 2. 